\title{
Longitudinal exploration of in situ mock code events and the performance of cardiac arrest skills
}

\author{
Samuel Owen Clarke, ${ }_{1}^{1}$ Ian M Julie, ${ }^{1}$ Aubrey P Yao, ${ }^{2}$ Heejung Bang, ${ }^{3}$ Joseph D Barton, ${ }^{4}$ \\ Sameerah M Alsomali, ${ }^{5}$ Matthew V Kiefer, ${ }^{6}$ Ali Hassan Al Khulaif, ${ }^{7}$ Muna Aljahany, ${ }^{8}$ \\ Sandhya Venugopal, ${ }^{9}$ Aaron E Bair ${ }^{1}$
}

'Department of Emergency Medicine, University of

California, Davis, Sacramento, California, USA

2Department of Anesthesia, University of California, Davis, Sacramento, California, USA

${ }^{3}$ Division of Biostatistics, University of California, Davis, Sacramento, California, USA ${ }^{4}$ Department of Emergency Medicine, Kaiser Permanente Antioch Medical Center, Antioch, California, USA ${ }^{5}$ Department of Emergency Medicine, King Saud Bin Abdulaziz University for Health Sciences, Riyadh, Saudi Arabia ${ }^{6}$ Emergency Medicine, St. Joseph's Medical Center, Stockton, California, USA ${ }^{7}$ Emergency Medicine, Prince Sultan Military Medical City, Riyadh, Saudi Arabia

${ }^{8}$ Emergency Medicine, Princess Nourah Bint Abdulrahman University, Riyadh, Riyadh, Saudi Arabia

${ }^{9}$ Division of Cardiology, University of California, Davis, Sacramento, California, USA

\section{Correspondence to} Dr Samuel Owen Clarke, Department of Emergency Medicine, University of California, Davis, Sacramento, CA 95616, USA; soclarke@ ucdavis.edu

Received 14 September 2017 Revised 12 February 2018 Accepted 13 February 2018 Published Online First 2 March 2018

Check for updates

To cite: Clarke SO, Julie IM, Yao AP, et al. BMJ Stel 2019:5:29-33.

\begin{abstract}
Introduction In-hospital cardiac arrest (IHCA) affects 200000 adults in the USA each year, and resuscitative efforts are often suboptimal. The objective of this study was to determine whether a programme of 'mock codes' improves group-level performance of IHCA skills. Our primary outcome of interest was change in cardiopulmonary resuscitation (CPR) fraction, and the secondary outcomes of interest were time to first dose of epinephrine and time to first defibrillation. We hypothesised that a sustained programme of mock codes would translate to greater than 10\% improvement in each of these core metrics over the first 3 years of the programme.
\end{abstract}

Methods We conducted mock codes in an urban teaching hospital between August 2012 and October 2015. Mock codes occurred on Telemetry and Medical/ Surgical units on day and night shifts. Codes were managed by unit staff and members of the hospital's 'Code Blue' team, and data were recorded by trained observers. Data were summarised using descriptive statistics, and repeated measures outcomes were calculated using a mixed effects model.

Results Fifty-seven mock codes were included in the analysis: 42 on Medical/Surgical units and 15 on Telemetry units. CPR fraction increased by $2.9 \%$ per 6-month time interval on Telemetry units, and $1.3 \%$ per time interval on Medical/Surgical units. Neither time to first epinephrine dosing nor time to defibrillation changed significantly.

Conclusions While we observed a significant improvement in CPR fraction over the course of this programme of mock codes, similar improvements were not observed for other key measures of cardiac arrest performance.

\section{INTRODUCTION}

Survival from in-hospital cardiac arrest (IHCA) depends on the coordinated efforts of multiple providers. ${ }^{1}$ While both patient factors (eg, age, comorbidities) and circumstantial factors (eg, initial rhythm, witnessed arrest) play a substantial role in determining the likelihood of survival, factors intrinsic to the process of resuscitation do as well. ${ }^{2}$ Cardiopulmonary resuscitation (CPR) fraction (the proportion of time that a pulseless patient receives $\mathrm{CPR}$ ), time to defibrillation for shockable rhythms and the timing of vasoactive medications (eg, epinephrine) in relation to the onset of cardiac arrest have all been shown to be predictive of survival. ${ }^{3-7}$ These factors rest largely in the hands of rescuers who respond to IHCA events.

The education and training of hospital staff who respond to IHCA events are therefore essential to improving outcomes. ${ }^{89}$ However, training for IHCA poses multiple challenges to hospitals, as IHCA events are relatively infrequent and the composition of staff responding to those events changes frequently. ${ }^{8}$ The traditional paradigm of periodic, classroom-based training has repeatedly been shown to fall short of providing durable and accurate knowledge and skills among hospital staff who respond to cardiac arrest. ${ }^{10-12}$ As a result, physician house staff have reported feeling unprepared to manage IHCA events, ${ }^{13}$ and resuscitative efforts often fall short of published guidelines. ${ }^{14}$

Simulated IHCA events or mock codes have shown promise as a means of providing realistic team training for hospital staff. Recent studies have demonstrated improved adherence to IHCA guidelines by rescuers as well as improved survival from IHCA among children. ${ }^{15} 16$ However, these studies have focused on short-term training interventions and individual practice environments (eg, paediatric ICU). The utility of mock codes as a means of increasing institution-wide readiness for responding to IHCA has been inadequately explored.

The objective of this study was to determine whether a hospital-wide campaign of simulated 'mock codes' leads to improvements in objective measures of group-level performance of cardiac resuscitation skills over time at a single academic hospital. The primary outcome of interest was improvement in CPR fraction, and the secondary outcomes of interest were time to first dose of epinephrine and time to first defibrillation. We hypothesised that a sustained programme of mock codes would translate to greater than $10 \%$ improvement in each of these core metrics over the course of the first 3 years the programme has been in place.

\section{METHODS \\ Study design}

This was a prospective study of in situ 'mock codes' held within a single teaching hospital in northern California between 23 August 2012 and 12 October 2015.

\section{Setting and participants}

UC Davis Medical Center is a Level I Trauma centre with an annual patient volume of 79500 Emergency Department visits and 33000 hospital admissions. 
IHCA events are managed by a multidisciplinary group of 'Code Blue' responders including unit staff, medical intensive care unit (MICU) and rapid response team nurses, pharmacists, respiratory therapists and physician trainees (residents and fellows) from anesthesiology, surgery, internal medicine and emergency medicine services. Physician house staff from multiple services (internal medicine, emergency medicine, anesthesiology) rotate through our MICU on 1 month rotations and participate on the Code Blue team during their rotations.

\section{Mock codes}

As part of a multidisciplinary training initiative that began in August 2012, simulated 'mock codes' were held on Medical/ Surgical and Telemetry nursing units 2-3 times per month throughout the hospital. During the study period, mock codes were conducted on both day and night shifts. For each mock code, a nursing staff member was recruited immediately prior to the event to act as a 'primary nurse.' This nurse was given a description of a patient awaiting initial assessment after being admitted to the unit, as well as background information including medical history and relevant data (eg, imaging and lab work). The nurse would find the patient pulseless and apneic, and a 'Code Blue Drill' would be called on the nurse's prompting via the hospital's overhead paging system. The code was then responded to and managed by the hospital's Code Blue response team and local nursing unit staff. Two scenarios were used: one in which an adult male patient experienced a pulseless electrical activity arrest secondary to opiate-induced hypoventilation, and one in which an adult male patient experienced a pulseless ventricular tachycardia arrest after being admitted for pneumonia. We designed our simulation cases to reflect variations in pathophysiology that come into play when managing cardiac arrest and to highlight scenarios that are of clinical relevance in the hospital setting. Simulations were performed using a Laerdal 3G human patient simulator (Laerdal Medical, Wappingers Falls, New York, USA).

\section{Data collection}

During the study period, mock codes were held on Telemetry and Medical/Surgical nursing units throughout the hospital on both day and night shifts. Staff were not given prior notice that a mock code was to occur. Trained research assistants were present in the room for each code and completed a structured data collection form which was used to measure all of the time-dependent outcomes in the study.

\section{Data analysis}

CPR fraction was calculated by dividing the cumulative time that the manikin received chest compressions by the total pulseless time (time from initiation of CPR until verbal confirmation of return of pulses). Time 'zero' was defined either (1) verbal recognition by a participant that the simulated patient was in cardiac arrest or (2) initiation of chest compressions. Mock codes were stratified into 6-month time intervals (designated one through seven as time configuration) by date for analysis comparison. Data from the mock codes were summarised using descriptive statistics, including sample size/number of observations, mean and SD. We compared Telemetry units to Medical/Surgical units due to the potential differences in training among staff (those on Telemetry units in our hospital have mandatory advanced cardiac life support (ACLS) training, whereas staff on Medical/ Surgical units are only required to have basic life support (BLS) training) as well as the frequency of events (cardiac arrest being

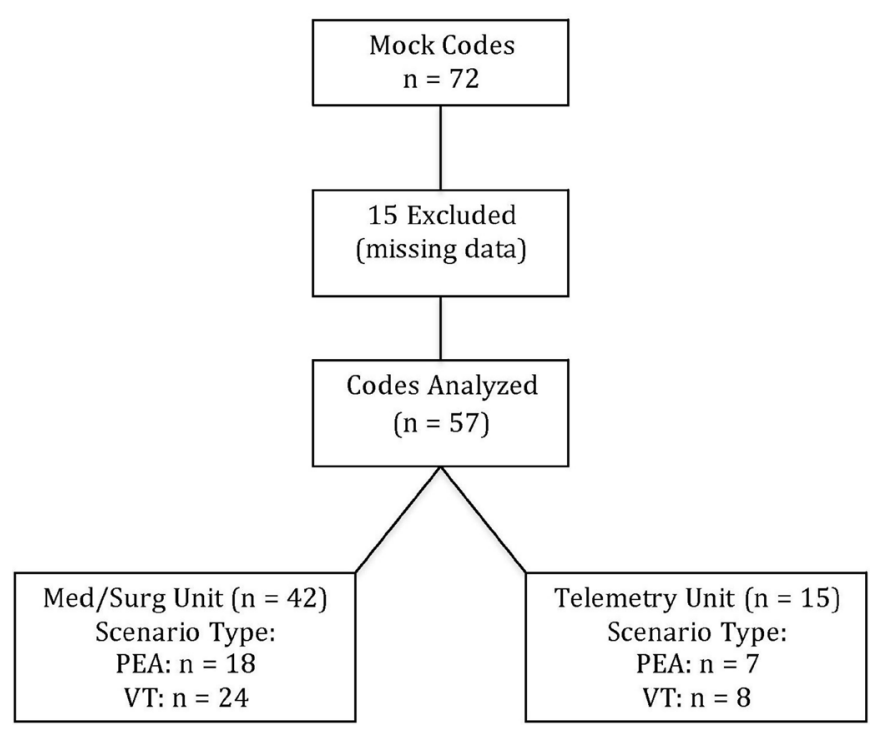

Figure 1 Study enrolment by unit type (Medical/Surgical and Telemetry) and simulation scenario (pulseless electrical activity and ventricular tachycardia).

a more common occurrence on units to which patients have been admitted specifically for cardiac monitoring). Mixed effects models were fitted to account for correlation among longitudinal measurements of the outcomes within the same unit with three covariates: unit, time and unit"time interaction, where compound symmetry covariance was used and time was coded by interval $1-7 . .^{17}$ Pearson (interclass) correlation and intraclass correlation coefficients (ICC) were calculated for mock codes in subsamples $(n=11)$ in which more than one observer was present, for reliability assessment. SAS V.9.4 was used for statistical analysis. Since there was a single primary outcome, we did not adjust the $\mathrm{p}$ value for multiple testing.

\section{RESULTS}

During the study period, there were 72 mock codes. Fifty-seven codes were included in the analysis, and 15 were excluded due to missing data (figure 1). Two observers were present for 11 codes $(11 / 52=21 \%)$, and their interobserver reliability for all outcomes was high (ICC and Pearson correlation coefficient 0.79-0.98; table 1).

The average CPR fraction for Med/Surg units across all time periods was 0.75 ( $\mathrm{SD}=0.09$; from $\mathrm{n}=42$ observations) and for Telemetry units was $0.72(\mathrm{SD}=0.12 ; \mathrm{n}=15)$ (table 2$)$.

The average time to epinephrine delivery was 417 s $(\mathrm{SD}=122$; $\mathrm{n}=42)$ for Med/Surg units and $375 \mathrm{~s}(\mathrm{SD}=97 ; \mathrm{n}=15)$ for Telemetry units. The average time to defibrillation for codes with a shockable rhythm was $413 \mathrm{~s}(\mathrm{SD}=172 ; \mathrm{n}=22)$ for $\mathrm{Med} / \mathrm{Surg}$ units and $312 \mathrm{~s}(\mathrm{SD}=95 ; \mathrm{n}=8)$ for Telemetry units.

CPR fraction increased $2.9 \%$ per time interval on Telemetry units ( $\mathrm{p}$ value $=0.04$ ) and $1.34 \%$ per time interval on Medical/ Surgical units) (table $3 \mathrm{~A}$, figure 2).

The difference in change in CPR fraction between Medical/ Surgical and Telemetry units was not significant ( $p$ value $=0.36$ ). When we modelled the main effects of unit and time (without the interaction term which is not statistically significant), the overall time trend common slope was $1.8 \%$ per time interval ( $\mathrm{p}$ value $=0.02$; results not shown in tables). Time trend as well as differential time trend for different units were not statistically significant for the two secondary outcomes ( $p$ value $>0.16$, table 3B,C); see figures 2-4 for time trends. 
Table 1 Results of inter-rater reliability analysis for mock codes $(n=11)$

\begin{tabular}{|c|c|c|c|c|c|}
\hline Mean & SD & Minimum & Maximum & $\begin{array}{l}\text { Pearson correlation } \\
\text { coefficient }\end{array}$ & $\begin{array}{l}\text { Intraclass correlation } \\
\text { coefficient }\end{array}$ \\
\hline \multicolumn{6}{|c|}{ (A) CPR interruptions } \\
\hline 19.0 & 6.5 & 6 & 28 & \multirow[t]{2}{*}{0.89} & \multirow[t]{2}{*}{0.90} \\
\hline 18.7 & 6.8 & 5 & 27 & & \\
\hline \multicolumn{6}{|c|}{ (B) CPR time } \\
\hline 428.4 & 108.5 & 257 & 690 & \multirow[t]{2}{*}{0.98} & \multirow[t]{2}{*}{0.98} \\
\hline 422.5 & 106.7 & 277 & 698 & & \\
\hline \multicolumn{6}{|c|}{ (C) CPR fraction } \\
\hline 0.68 & 0.14 & 0.36 & 0.86 & \multirow[t]{2}{*}{0.98} & \multirow[t]{2}{*}{0.98} \\
\hline 0.68 & 0.14 & 0.39 & 0.86 & & \\
\hline \multicolumn{6}{|c|}{ (D) Time to epinephrine administration } \\
\hline 441 & 153 & 200 & 747 & \multirow[t]{2}{*}{0.82} & \multirow[t]{2}{*}{0.79} \\
\hline 414 & 116 & 189 & 613 & & \\
\hline
\end{tabular}

Intraclass correlation coefficient was estimated from mixed effects model.

CPR, cardiopulmonary resuscitation.

\section{DISCUSSION}

During the study period, we observed a significant longitudinal improvement in CPR fraction during mock codes on both Medical/Surgical and Telemetry units. Despite this observed improvement, CPR fraction for all time periods and unit types was consistently below the recommended goal of $80 \% .{ }^{18}$ It is interesting to note that there was an apparent decrease in CPR fraction during time interval 6 (figure 2). This does not appear to have been a 'July effect' of staff turnover in a teaching hospital, as this time interval occurred in January-July 2015 (ie, the second half of the academic cycle). It also does not appear to reflect the influence of simulation scenario type, as the ventricular tachycardia scenario was introduced in January 2014 and the subsequent time interval (interval 4) showed a relatively high CPR fraction. We suspect that this finding reflects the nature of an institution-wide intervention in which gains may happen in a non-linear fashion. Neither time to epinephrine nor time to defibrillation changed significantly for either unit type during the study period. Taken together, our results suggest an uneven benefit to the practice of IHCA skills from in situ simulation.

A number of studies have described the conundrum of retaining the necessary knowledge and psychomotor skills needed for recommended IHCA management. These skills are most frequently taught in the USA through the American Heart Association's (AHA's) BLS and ACLS courses, ${ }^{19}$ which have served as the community standard for more than four decades. Whereas the interval for recertification in these courses is every 2 years, a systematic review by Yang suggested that both knowledge and psychomotor skills pertaining to cardiac arrest resuscitation decay significantly within $6-12$ months of training. ${ }^{12}$ The AHA has seemingly acknowledged that the traditional training interval of 2 years for ACLS and BLS courses is inadequate for the needs of hospital staff who may encounter IHCA on an

Table 2 CPR fraction, time to epinephrine and time to defibrillation averaged over time, by unit type

\begin{tabular}{lcl}
\hline & $\begin{array}{l}\text { Medical/Surgical unit } \\
\text { mean (SD), } \mathrm{n}\end{array}$ & $\begin{array}{l}\text { Telemetry unit mean } \\
\text { (SD), } \mathrm{n}\end{array}$ \\
\hline CPR fraction & $0.75(0.09), \mathrm{n}=42$ & $0.72(0.12), \mathrm{n}=15$ \\
Time to epinephrine (in s) & $417(122), \mathrm{n}=42$ & $375(97), \mathrm{n}=15$ \\
Time to defibrillation (in s) & $413(172), \mathrm{n}=22$ & $312(95), \mathrm{n}=8$ \\
\hline
\end{tabular}

$\mathrm{CPR}$, cardiopulmonary resuscitation; $\mathrm{n}$, sample size. infrequent basis. In a 2013 consensus statement, 'Strategies for Improving Survival After In-Hospital Cardiac Arrest in the United States,' the AHA has recommended that hospitals engage in training interventions such as mock codes to support staff readiness for these events. ${ }^{8}$

Simulation training is associated with greater adherence to ACLS guidelines among residents engaged in cardiac arrest response in comparison to those who have received traditional training. ${ }^{15}$ Better adherence to recommendations may in turn lead to greater chances of survival from IHCA events. ${ }^{20}$ Yet, processes of care such as performing high-quality CPR during resuscitation require that IHCA responders not only possess the necessary psychomotor skills but can also perform them well as a team. Leadership and the process of team building heavily influence key processes such as CPR fraction during an IHCA. ${ }^{21-25}$ We selected this as our primary outcome measure as it reflects the overall contribution of these various inputs to an objective and clinically meaningful yardstick of resuscitation performance.

The field of human factors research has provided key insights to the nature of complex systems such as hospitals, and the factors which contribute to suboptimal performance. ${ }^{26}$ IHCA presents a conundrum in that issues at the level of individual providers (eg, prior training and experience) as well as the institution (eg,

Table 3 Results of mixed effects model describing changes over time in CPR fraction, time to epinephrine administration and time to defibrillation

\begin{tabular}{|c|c|c|c|}
\hline Factor & Estimate & SE & $P$ values \\
\hline \multicolumn{4}{|c|}{ (A) CPR fraction: primary outcome } \\
\hline Med/Surg (vs Telemetry) & 0.096 & 0.07 & 0.23 \\
\hline Time & 0.029 & 0.01 & 0.04 \\
\hline Time*Med/Surg & -0.015 & 0.02 & 0.36 \\
\hline \multicolumn{4}{|l|}{ (B) Time to epinephrine } \\
\hline Med/Surg (vs Telemetry) & -75.1 & 91.3 & 0.43 \\
\hline Time & -11.3 & 18.1 & 0.54 \\
\hline Time*Med/Surg & 30.1 & 21.4 & 0.17 \\
\hline \multicolumn{4}{|l|}{ (C) Time to defibrillation } \\
\hline Med/Surg (vs Telemetry) & 515.1 & 317 & 0.14 \\
\hline Time & 45.4 & 49.6 & 0.37 \\
\hline Time*Med/Surg & -79.6 & 59.0 & 0.19 \\
\hline
\end{tabular}

$\mathrm{CPR}$, cardiopulmonary resuscitation. 


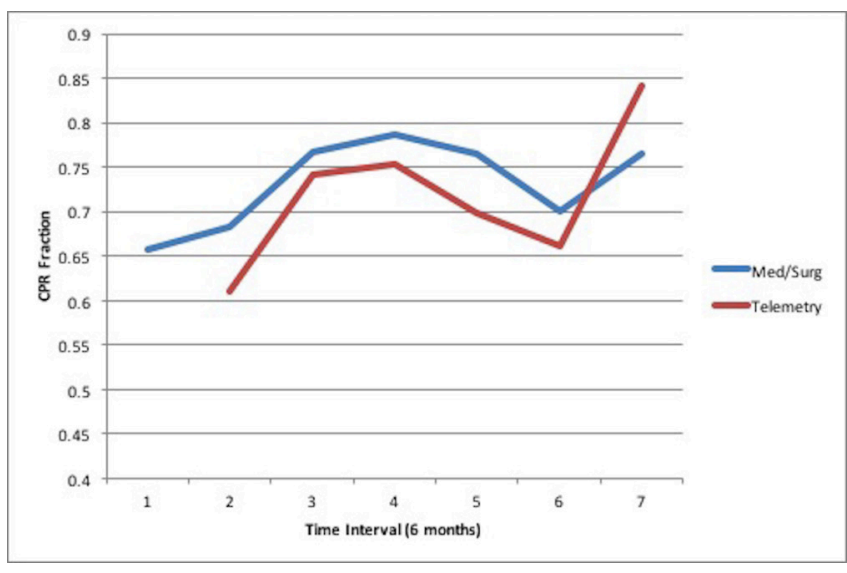

Figure 2 Changes in cardiopulmonary resuscitation (CPR) fraction over time and by unit type.

organisation of cardiac arrest response team) may facilitate or hinder resuscitation. Team performance relies on mutual understanding of roles and responsibilities, clear and direct communication, and efficient distribution of tasks, ${ }^{21}$ all of which are more easily achieved by dedicated teams rather than teams which form ad hoc. ${ }^{1}$ However, even hospitals which utilise dedicated code teams may suffer from delays in response to IHCA events. ${ }^{1} 27$ The skills of local unit staff who act as first responders to an IHCA event are therefore critical to establishing the first steps of resuscitation. $^{27}$

We believe that it is in practising for this first $5 \mathrm{~min}$ of IHCA response that mock codes find their most important role. This is the time period in which unit staff can establish the essential processes of cardiac arrest care that will ultimately have the greatest impact on patient outcomes: effective chest compressions, bag-valve mask ventilation, intravenous access and defibrillator deployment. Establishing this 'base layer' of resuscitation may help to tighten the interval of time between recognition of cardiac arrest and first defibrillation and cardioactive drug administration once ACLS-trained providers arrive.

While our study failed to show statistically significant improvement in time to defibrillation and time to first epinephrine during mock codes, we suspect that this may reflect a latent threat in the form of lag time between initiation of the IHCA response by unit staff and the arrival of the Code Blue team. While nursing staff on Telemetry units in our hospital are ACLS

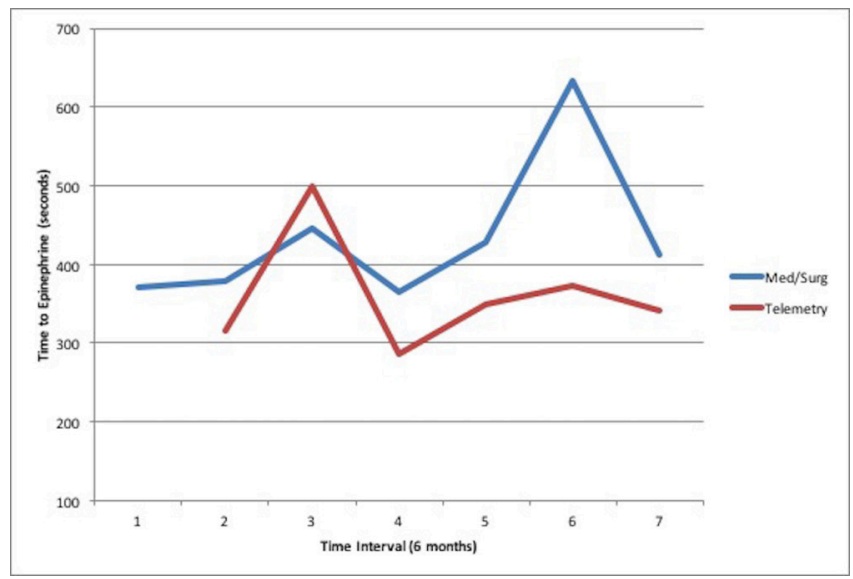

Figure 3 Changes in time to epinephrine administration over time and by unit type.

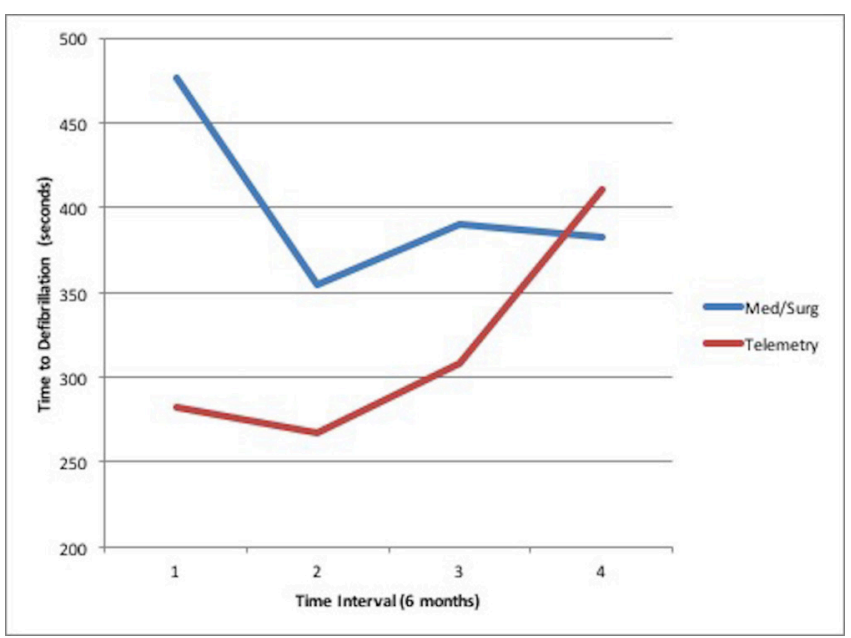

Figure 4 Changes in time to defibrillation over time and by unit type.

trained, they may feel hesitant to defibrillate patient or administer cardioactive medications prior to the arrival of a physician code team leader. This may lead to further delays as the Code Blue team orients itself to the situation and organises itself. As a result, we have begun augmenting in situ mock codes with additional training for nursing unit staff and code team members. Our rapid response team conducts on site 'first responder' training that focuses on the first few minutes of IHCA resuscitation, and our simulation centre staff holds quarterly training workshops for our code team. These workshops focus on aspects of leadership, teamwork and decision-making relevant to ICHA resuscitation.

While our study assessed the impact of an increasingly utilised training intervention (mock codes) on a recognised indicator of resuscitation performance (CPR fraction), extrapolation from these data to actual patients and patient-centred outcomes is speculative. The study measured group-level performance among staff responding to mock codes and did not control for bias from individual staff members who may have participated in multiple codes. The study intervention was carried out on Medical/ Surgical and Telemetry units throughout the hospital and was not powered to measure changes in performance on individual units over time. The training intervention also employed two different simulation cases (a pulseless electrical activity case and a ventricular tachycardia case) which may have influenced the behaviour of participants.

Despite these limitations, we believe that a number of important conclusions can be derived from this study. While mock codes may play an important role in an institution's readiness strategy for IHCA management, they should not be viewed as a stand-alone intervention. We have found them to be of particular benefit to unit staff who participate in codes infrequently and useful as a means for practising the essential skills of teamwork and communication between staff as they might play out in an actual IHCA event. However, a sustained campaign of mock codes poses a number of logistical challenges. Providing the training experience throughout a hospital and on day and nighttime shifts requires a significant contribution of training resources, and finding adequate time and space for hospital staff to participate in mock codes in the era of ever-increasing hospital censuses is itself quite challenging.

\section{CONCLUSIONS}

Our study has demonstrated the positive impact of mock codes on CPR fraction, a key performance measure for cardiac arrest 
resuscitation. However, this training benefit did not extend to other key elements of the resuscitation process. Institutions using mock codes for IHCA training should bear these findings in mind and should view them as an adjunct to more focused training.

Contributors $\mathrm{SOC}, \mathrm{IMJ}, \mathrm{HB}, \mathrm{APY}$ and $\mathrm{AEB}$ contributed to the origination and design of this study. SOC, IMJ, APY, JDB, SMA, MVK, AHAK, MA and SV were responsible for the data collection. SOC, IMJ, HB and APY contributed to the data analysis and interpretation of the study. All the authors contributed to the writing and critical review of this manuscript.

Funding The efforts of HB for this study were supported in part by the National Center for Advancing Translational Sciences, National Institutes of Health, through grant number UL1 TR001860.

Competing interests None declared.

Ethics approval UC Davis Institutional Review Board.

Provenance and peer review Not commissioned; externally peer reviewed.

(c) Article author(s) (or their employer(s) unless otherwise stated in the text of the article) 2019. All rights reserved. No commercial use is permitted unless otherwise expressly granted.

\section{REFERENCES}

1 Hunziker S, Tschan F, Semmer NK, et al. Hands-on time during cardiopulmonary resuscitation is affected by the process of teambuilding: a prospective randomised simulator-based trial. BMC Emerg Med 2009;9:3.

2 Marsch SC, Müller C, Marquardt K, et al. Human factors affect the quality of cardiopulmonary resuscitation in simulated cardiac arrests. Resuscitation 2004:60:51-6.

3 Andersen LW, Kurth T, Chase M, et al. Early administration of epinephrine (adrenaline) in patients with cardiac arrest with initial shockable rhythm in hospital: propensity score matched analysis. BMJ 2016 353:11577.

4 Idris AH, Guffey D, Aufderheide TP, et al. Relationship between chest compression rates and outcomes from cardiac arrest. Circulation 2012;125:3004-12.

5 Christenson J, Andrusiek D, Everson-Stewart S, et al. Chest compression fraction determines survival in patients with out-of-hospital ventricular fibrillation. Circulation 2009;120:1241-7.

6 Chan PS, Krumholz HM, Nichol G, et al. Delayed time to defibrillation after in-hospital cardiac arrest. N Eng/ J Med Overseas Ed 2008;358:9-17.

7 Donnino MW, Andersen LW, Berg KM, et al. Temperature management after cardiac arrest: an advisory statement by the advanced life support task force of the international liaison committee on resuscitation and the american heart association emergency cardiovascular care committee and the council on cardiopulmonary, critical care, perioperative and resuscitation. Resuscitation 2016:98:97-104.

8 Morrison LJ, Neumar RW, Zimmerman JL, et al. Strategies for improving survival after in-hospital cardiac arrest in the United States: 2013 consensus recommendations: a consensus statement from the American Heart Association. Circulation 2013;127:1538-63.
9 Moretti MA, Cesar LA, Nusbacher A, et al. Advanced cardiac life support training improves long-term survival from in-hospital cardiac arrest. Resuscitation 2007;72:458-65

10 Young R, King L. An evaluation of knowledge and skill retention following an in-house advanced life support course. Nurs Crit Care 2000;5:7-14.

11 Smith KK, Gilcreast D, Pierce K. Evaluation of staff's retention of ACLS and BLS skills. Resuscitation 2008;78:59-65.

12 Yang CW, Yen ZS, McGowan JE, et al. A systematic review of retention of adult advanced life support knowledge and skills in healthcare providers. Resuscitation 2012;83:1055-60.

13 Hayes CW, Rhee A, Detsky ME, et al. Residents feel unprepared and unsupervised as leaders of cardiac arrest teams in teaching hospitals: a survey of internal medicine residents. Crit Care Med 2007:35:1668-72.

14 Abella BS, Sandbo N, Vassilatos P, et al. Chest compression rates during cardiopulmonary resuscitation are suboptimal: a prospective study during in-hospital cardiac arrest. Circulation 2005;111:428-34.

15 Wayne DB, Didwania A, Feinglass J, et al. Simulation-based education improves quality of care during cardiac arrest team responses at an academic teaching hospital: a case-control study. Chest 2008:133:56-

16 Andreatta P, Saxton E, Thompson M, et al. Simulation-based mock codes significantly correlate with improved pediatric patient cardiopulmonary arrest survival rates. Pediatr Crit Care Med 2011;12:33-8.

17 Fitzmaurice GM, Laird NM, Ware JH. Applied longitudinal analysis. New Jersey, USA: John Wiley \& Sons, 2012.

18 Meaney PA, Bobrow BJ, Mancini ME, et al. Cardiopulmonary resuscitation quality: [corrected] improving cardiac resuscitation outcomes both inside and outside the hospital: a consensus statement from the American Heart Association. Circulation 2013:128:417-35.

19 Link MS, Berkow LC, Kudenchuk PJ, et al. Part 7: adult advanced cardiovascular life support. Circulation 2015;132:S444-S464.

20 McEvoy MD, Field LC, Moore HE, et al. The effect of adherence to ACLS protocols on survival of event in the setting of in-hospital cardiac arrest. Resuscitation 2014:85:82-7.

21 Andersen PO, Jensen MK, Lippert A, et al. Identifying non-technical skills and barriers for improvement of teamwork in cardiac arrest teams. Resuscitation 2010;81:695-702.

22 Hunziker S, Bühlmann C, Tschan F, et al. Brief leadership instructions improve cardiopulmonary resuscitation in a high-fidelity simulation: a randomized controlled trial. Crit Care Med 2010:38:1086-91

23 Hunziker S, Johansson AC, Tschan F, et al. Teamwork and leadership in cardiopulmonary resuscitation. J Am Coll Cardiol 2011;57:2381-8.

24 Yeung JH, Ong GJ, Davies RP, et al. Factors affecting team leadership skills and their relationship with quality of cardiopulmonary resuscitation. Crit Care Med 2012:40:2617-21.

25 Krage R, Zwaan L, Tjon Soei Len L, et al. Relationship between non-technical skills and technical performance during cardiopulmonary resuscitation: does stress have an influence? Emerg Med I 2017;34:728-33.

26 Reason J. Understanding adverse events: human factors. Qual Health Care 1995;4:80-9.

27 Hunt EA, Walker AR, Shaffner DH, et al. Simulation of in-hospital pediatric medical emergencies and cardiopulmonary arrests: highlighting the importance of the first 5 minutes. Pediatrics 2008;121:e34-e43. 